\title{
Spontaneously Reported Adverse Reactions for Herbal Medicinal Products and Natural Remedies in Sweden 2007-15: Report from the Medical Products Agency
}

\author{
Erika Svedlund $^{1} \cdot$ Maria Larsson $^{2} \cdot$ Robert Hägerkvist $^{1}$ (1)
}

Published online: 28 March 2017

(c) The Author(s) 2017. This article is an open access publication

\begin{abstract}
Background In relation to the extensive use of herbal medicinal products in self-care, the safety information is limited and there is a need for improvement. This study describes spontaneously reported adverse reactions related to herbal medicinal products and natural remedies in Sweden.

Objectives To evaluate the characteristics and frequency of adverse events recorded by the Swedish Medical Products Agency, where herbal medicinal products and natural remedies were suspected as causative agents.

Methods Adverse drug reactions reported to the Swedish Medical Product Agency during 2007-15 related to approved herbal medicinal products or natural remedies were included and analysed in the retrospective study. Reports had been assessed for causality when they were lodged and only reports that had been assessed as at least possible were included in the study.

Results In total, 116 reports (concerning 259 adverse reactions) related to herbal medicinal products or natural remedies were found in the Swedish national pharmacovigilance database. The active ingredients most frequently suspected during the study period were black
\end{abstract}

Erika Svedlund and Robert Hägerkvist contributed equally to this article.

Electronic supplementary material The online version of this article (doi:10.1007/s40801-017-0104-y) contains supplementary material, which is available to authorized users.

Robert Hägerkvist

robert.hagerkvist@mpa.se

1 Department of Herbal Medicinal Products, Medical Products Agency, P.O. Box 26, SE-751 03 Uppsala, Sweden

2 Department of Pharmacovigilance, Medical Products Agency, Uppsala, Sweden cohosh rhizome (15 reports), purple coneflower herb (14 reports) and a combination of extracts of pollen (13 reports). Adverse reactions related to skin and subcutaneous tissue were the most commonly reported reactions. Conclusions No previously unknown safety problems have been discovered in the present study. This finding could be explained by a thorough pre-approval assessment of medicinal products and the fact that most herbal preparations in medicinal products have been in clinical use for many years (for traditional herbal medicinal products, the requirements are $\geq 30$ years), i.e. adverse reactions are acknowledged and assessed before approval.

\section{Key Points}

The most commonly reported adverse reactions for herbal medicinal products and natural remedies were related to skin and subcutaneous tissue. These reactions could be the result of hypersensitivity and can be viewed as an inherent problem with herbal treatments.

In relation to the extensive use of herbal medicinal products in self-care, the safety information is limited. Healthcare professionals and patients are encouraged to discuss the use of and report suspected adverse reactions related to herbal medicinal products.

\section{Introduction}

Medicinal products included in the study, with the active ingredient of herbal origin, are approved according to the current European Directive 2001/83/EC. These products 
can be approved as herbal medicinal products (HMPs) or as traditional herbal medicinal products (THMPs). In Sweden, there is also a national law allowing approved natural remedies (NRs) with active ingredients of natural origin that are of non-herbal origin, i.e. consists of an animal part, a bacterial culture, a mineral or a salt [1]. Natural remedies are always intended for self-care. All three categories, HMPs, THMPs and NRs, have the same requirements regarding the quality of the product as any other medicinal product. The main distinction between HMPs and THMPs is that the efficacy requirements for THMPs consist solely of long-standing medicinal use (15 years within the European Economic Area and 30 years worldwide). In addition, THMPs are strictly limited to self-medication that does not require a physician's diagnosis, prescription or supervision of treatment. The THMPs are only intended for oral administration, external application and/or inhalation. A recent publication provides the details of the requirements for HMPs and THMPs in the European Union [2].

In relation to the widespread use of HMPs, THMPs and NRs in self-care, the safety information from actual use is limited and there is a constant demand for increasing adverse drug reaction (ADR) reporting. We believe that analysing pharmacovigilance data, i.e. ADR reports, is one approach to increase the awareness in this important area. Therefore, in the present study we investigated the pattern of ADRs related to approved medicinal products of natural origin (HMPs, THMPs and NRs) in Sweden.

Our study is a follow-up on the study by Jacobsson et al., which investigated the pattern of spontaneously reported adverse reactions related to complementary and alternative medicinal (CAM) products in Sweden over two decades, between 1987 and 2006 [3]. Our study followed all spontaneously reported ADRs of HMPs in Sweden over almost another decade, between 1 January, 2007 and 31 December, 2015. During this period, the average number of HMPs, THMPs and NRs in Sweden each year was approximately 100 products, mainly over-the-counter products and they account for approximately $10 \%$ of the over-the-counter products on the Swedish market.

There is one important difference between the present study and the previous study. The previous study by Jacobsson et al. [3] included CAM products in a broader sense, i.e. reports of adverse reactions related to food supplements were also included. During the time period of that study, reports related to food supplements were regularly sent to the Swedish Medical Products Agency (MPA) and included in the Swedish pharmacovigilance database. However, food supplements do not fall under the pharmacovigilance legislation and adverse reactions related to the intake of food supplements should now be reported to the National Food Agency in Sweden. For the last few years, reports related to food supplements have not been available in the MPA pharmacovigilance database and are not included in this study. Hence, the MPA can only monitor the safety of approved medicinal products. Therefore, the aim of this report is to evaluate the characteristics and frequency of adverse events recorded by the MPA, where HMPs, THMPs and NRs were suspected as causative agents.

\section{Methods}

All spontaneously reported ADRs to the Swedish MPA between 1 January, 2007 and 31 December, 2015, where at least one medicinal product was categorised as a HMP, a THMP or as a NR that was suspected to cause the ADR, were included in this retrospective study.

Each assessed ADR report contains information about the patient, the ADR, and the treatment suspected to have caused it and concomitant drugs. Concomitant drugs presented in this study include both suspected and non-suspected drugs. No causality assessment is presented for concomitant drugs.

A report can contain one or more ADRs connected to one or more treatments. This information, as well as the MPA's causality assessment, are stored in the Swedish national pharmacovigilance database. All ADRs reported to the MPA are evaluated and a causality assessment is added to the report. The causality assessment is made according to the World Health Organization's criteria (Supplementary Table 1). Only reports rated possible or higher were included in the study.

The reported adverse reactions are presented according to the MedDRA (Medical Dictionary for Regulatory Activities) classification. The MedDRA classification is divided into five levels of hierarchy ranging from a very specific term, i.e. how it might be written in the report, to a system organ class. The adverse reactions in Supplementary Tables 2-5 are presented with the preferred term level, which is a single medical concept for a symptom (http:// www.meddra.org/how-to-use/basics/hierarchy).

The type of report is also categorised as serious or nonserious. The definition of a serious adverse reaction is an adverse reaction that results in death, is life-threatening, requires inpatient hospitalisation or prolongation of existing hospitalisation, results in persistent or significant disability or incapacity, or is a congenital anomaly/birth defect (Directive 2001/83/EC). The reporter may also set the case as other serious medical event. The remaining reports are considered non-serious. Every serious ADR report is assessed by a physician at the MPA.

From each included report, the suspected herbal preparation, the adverse reaction/s, the severity of the adverse reaction (i.e. serious/non-serious) and the source of the 
report are recorded. In this study, the source of the report is characterised as a healthcare professional (physician, nurse, pharmacist or dentist) or patient report. Reports from marketing authorisation (registration) holders are not included in the Swedish national pharmacovigilance database. Thus, duplicate reports do not occur.

\section{Results}

A total of 116 reports with 259 adverse reactions reported to be related to HMPs, THMPs or NRs were identified in the Swedish pharmacovigilance database during the study period. The numbers of reports per year during 2007-15 are shown in Fig. 1. The total number of

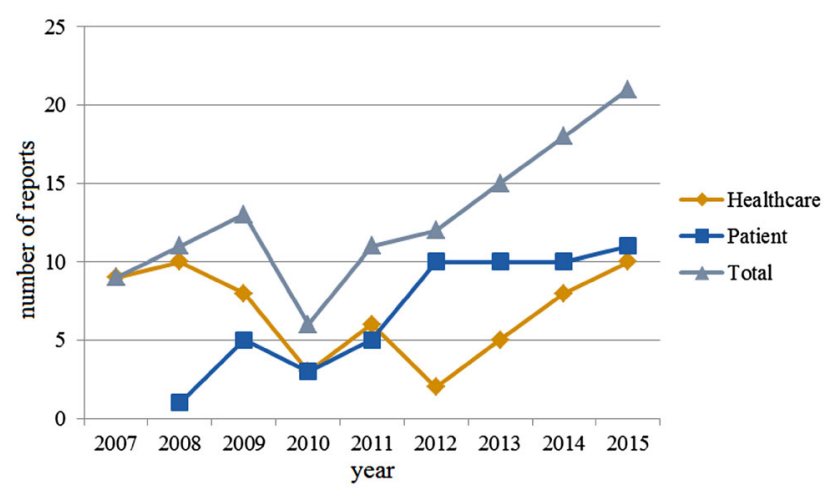

Fig. 1 Total number of reports per year for herbal medicinal products, traditional herbal medicinal products and natural remedies (filled triangles), number of reports per year from healthcare professionals (filled diamonds) and number of reports per year from patients (filled squares) reports per year varies between six and 21 reports. In addition, the number of reports per year from healthcare professionals and patients are presented in Fig. 1. The vast majority of reports (113/116) included in the study had been causality assessed as 'possible'. Three reports (3/116) were assessed as 'probable'. These three were non-serious reports from healthcare professionals. For each year since patient reporting was started, the MPA has received patient reports related to HMPs, THMPs, or NRs.

The total numbers of reports assessed as serious and non-serious from patients and healthcare professionals during the study period are shown in Table 1 . The most frequently suspected active substances and number of reports during the study period are also presented in Table 1. All other active substances had seven or fewer reports. For detailed data, all individual serious reports from healthcare professionals and patients are presented in Supplementary Tables 2 and 3, respectively. The individual non-serious reports from healthcare professionals and patients are presented in Supplementary Tables 4 and 5, respectively.

The interpretation of serious reactions was hampered by the fact that many of the reports contained concomitant medications or had low narrative quality (in the latter case, mainly patient reports). In an attempt to describe a typical serious reaction concerning the most frequently occurring plant materials, we summarise below three $(\mathrm{A}-\mathrm{C})$ of the healthcare reports related to the most frequently suspected herbal materials and with no concomitant medications mentioned.

A.A middle-aged woman experienced a pricking sensation in her throat, lips and tongue, approximately $2-3 \mathrm{~h}$ after intake of a lozenge containing purple coneflower.

Table 1 Serious and non-serious reports between 2007 and 2015 and the most frequently suspected active substances

Total number of reports assessed as serious and non-serious between 2007 and 2015

\begin{tabular}{|c|c|c|c|c|}
\hline Source of the report & Number of serious reports & \multicolumn{2}{|c|}{ Number of non-serious reports } & Total number of reports \\
\hline Healthcare professional & 21 & 40 & & 61 \\
\hline Patient & 13 & 42 & & 55 \\
\hline \multicolumn{5}{|c|}{ Most frequently suspected active substances between 2007 and 2015 (total number of reports, $n=116$ ) } \\
\hline \multicolumn{2}{|l|}{ Suspected substance } & $\begin{array}{l}\text { Number of } \\
\text { serious reports }\end{array}$ & $\begin{array}{l}\text { Number of non- } \\
\text { serious reports }\end{array}$ & $\begin{array}{l}\text { Total number } \\
\text { of reports }\end{array}$ \\
\hline \multicolumn{2}{|c|}{ Black cohosh rhizome (Cimicifuga racemosa L. Nutt.) } & 5 & 10 & 15 \\
\hline \multicolumn{2}{|c|}{$\begin{array}{l}\text { Purple coneflower herb, expressed juice } \\
\text { (Echinacea purpurea (L.) Moench) }\end{array}$} & 3 & 11 & 14 \\
\hline \multicolumn{2}{|c|}{$\begin{array}{l}\text { Pollen of maize, rye, cock's-foot, pine (Zea mays } \\
\text { L., Secale cereal L., Dactylis glomerata L., Pinus sylvestris L.) }\end{array}$} & 4 & 9 & 13 \\
\hline \multicolumn{2}{|c|}{$\begin{array}{l}\text { Gentian root, primula spp. flowers, sorrel spp. herb, elder flower, } \\
\text { verbena herb (Gentiana lutea L., Primula spp., Rumex } \\
\text { spp., Sambucus nigra L., Verbena officinalis L.) }\end{array}$} & 7 & 5 & 12 \\
\hline
\end{tabular}


Examination revealed redness on the throat. She was treated with corticosteroids and antihistamines. The condition gradually improved and the patient was discharged from hospital later the same day.

B.A previously healthy middle-aged woman presented at the emergency room with pain in the epigastrium, radiating to the right flank, and nausea. Her liver enzymes were considerably increased and continued to rise on her second day at the hospital and started to improve on the third day. She had begun self-medication with an HMP containing pollen of maize, rye, cock's foot and pine, approximately 2 weeks prior to the emergency room visit. Her medication was stopped and at follow-up 3 weeks later, her liver enzymes were almost normalised. The normalisation of the liver enzymes was interpreted as a positive de-challenge (Supplementary Table 1). Infection parameters were normal and there were no evidence for an autoimmune hepatitis. The reported diagnosis was a mixed liver reaction associated with the HMP.

C.A woman was experiencing a cold. After 1 day's use of an HMP containing gentian root, primula spp. flowers, sorrel spp. herb, elder flower and verbena herb, she developed a generalised urticaria. She self-medicated with antihistamines and went to sleep. The following morning she woke with swollen lips and face and a tingling sensation in one cheek. At the emergency room, she was treated with corticosteroids and antihistamines. A diagnosis of erythema multiforme was made. She recovered slowly and was discharged after a few days.

In Table 2, the most frequently reported adverse reactions are presented according to the MedDRA classification at the system organ class level. Similar to the previous publication [3], adverse reactions related to the skin and subcutaneous tissue were the most commonly reported reactions (Table 2).

\section{Discussion}

The main finding was that no previously unknown safety problems related to the use of HMPs have been discovered. Importantly, this conclusion is based upon high-quality data. All reports in the present study were assessed for causality and only reports rated as at least possible were included in the study. It takes fewer high-quality reports to form a basis for a signal and to understand the clinical relevance of the signal. Moreover, the regulatory system for medical products with a thorough assessment of the quality of the ingredients allows us to know with certainty which plant species and plant parts were implicated in the ADR. This is not always the case with herbal treatments, for example, in a systematic review of paediatric herbal adverse events from Gardiner et al., the implicated plant part was known only in $41 \%$ of the cases [4]. In the present study, the plant part is known in $100 \%$ of the cases.

In the present study, ADRs related to the skin and subcutaneous tissue are the most commonly reported reactions. This finding is in agreement with a previous study by Jacobsson et al. [3] and also in agreement with data from the World Health Organization [5]. Adverse skin and subcutaneous tissue reactions could be the result of hypersensitivity, including allergy, and can be viewed as an inherent problem with herbal treatments. Regulatory actions include appropriate labelling for medicinal products to minimise risks. A known hypersensitivity to the ingredients of an HMP is always a contraindication for use. This information is conveyed in the summary of product characteristics to healthcare professionals and to the patient in the product information leaflet. Unfortunately, not all patients are aware of their allergies or the possibility of cross-reactivity. Therefore, spontaneously reported ADRs related to hypersensitivity and allergies are expected to continue in a similar extent.

Table 2 Most frequently reported ADRs by SOC

\begin{tabular}{|c|c|c|}
\hline SOC & $\begin{array}{l}\text { Number of } \\
\text { reports }\end{array}$ & Suspected active substances with three or more ADRs (number of ADRs) \\
\hline $\begin{array}{l}\text { Skin and subcutaneous } \\
\text { tissue disorders }\end{array}$ & 32 & $\begin{array}{l}\text { Purple coneflower herb, expressed juice (7), gentian root, primula spp. flowers, sorrel spp. herb, } \\
\text { elder flower, verbena herb (6), black cohosh rhizome (4), valerian root (3), St John's wort (3) }\end{array}$ \\
\hline Gastrointestinal disorders & 29 & $\begin{array}{l}\text { Gentian root, primula spp. flowers, sorrel spp. herb, elder flower, verbena herb (4), thyme herb and } \\
\text { marshmallow root (4), valerian root (3) }\end{array}$ \\
\hline Investigations & 23 & $\begin{array}{l}\text { Black cohosh rhizome (7), Purple coneflower herb, expressed juice (3), pollen of maize, rye, } \\
\text { cock's-foot, pine (3), valerian root (3) }\end{array}$ \\
\hline General disorders & 21 & $\begin{array}{l}\text { Valerian root (4), pollen of maize, rye, cock's-foot, pine (3), purple coneflower herb, expressed } \\
\text { juice (3), black cohosh rhizome (3) }\end{array}$ \\
\hline Nervous system disorders & 20 & $\begin{array}{l}\text { Horse-chestnut seed (3), Arctic root root (3), pollen of maize, rye, cock's-foot, pine (3), St John's } \\
\text { wort (3) }\end{array}$ \\
\hline
\end{tabular}

$A D R s$ adverse drug reactions, SOC system organ class

a One report can contain one or more ADRs 
In the study by Jacobsson et al. [3], 35 cases of CAMdrug interaction causality assessed as 'possible' were reported. The most frequent interactions during this study period involved St John's wort. The interaction potential of St John's wort was observed and assessed by the Swedish MPA in the late 1990s. Today, the knowledge of the interaction potential of St John's wort is widespread among healthcare professionals and carefully communicated to patients in the product information leaflet. In the present study, there are no reports related to drug interactions and the use of St John's wort and only two reports related to drug interactions and the use of HMPs. These two reports on drug interaction concern the use of saw palmetto and an anticoagulant, dabigatran and warfarin, respectively. The dramatically reduced number of case reports on St John's wort interactions with other medicinal products could be explained by the increased awareness of the problem, which highlights the impact and importance of efficiently communicating safety signals.

Liver toxicity has been associated with the use of black cohosh, as discussed in the assessment report pertinent to the European Community herbal monograph on Cimicifuga racemosa (L.) Nutt., rhizoma [6, 7], where it is concluded that the available non-clinical and clinical data on liver toxicity associated with black cohosh are limited but the risk cannot be excluded and it is suggested that further case reports should be assessed thoroughly using the Roussel Uclaf Causality Assessment Method [8] to collect highquality data. Jacobsson et al. discuss in their study from 2008 [3] that several reports of different adverse liver reactions in subjects using black cohosh have been received by authorities in several countries, including six reports from Sweden (i.e. three reports on elevated transaminases and three reports on other various hepatic reactions). In the present retrospective study, five serious reports concerning black cohosh related to the liver. One report is from a patient (no laboratory values), the remaining four hospital reports unfortunately do not contain sufficient information for a Roussel Uclaf Causality Assessment Method-based analysis. The reports have been causality assessed only as possible and are confounded by co-medications. The absence of a Roussel Uclaf Causality Assessment Method and cofounding co-medications prevents any conclusion on liver toxicity associated with black cohosh in this study.

The number of spontaneously reported adverse reactions in this study is on average 13 reports per year. This number is much lower than the annual number of reports in the previous study by Jacobsson et al. [3], which reported an average of 39 reports annually. Interestingly, this outcome could be owing to the lack of reports related to nonmedicinal product (e.g. food supplements) in the present study. Unfortunately, the number of reports related to non- medicinal products in the Jacobsson et al. study is unknown, and a direct comparison is therefore not possible. It may be that either the majority of reports in the Jacobsson et al. [3] study concerned non-medicinal products, or that healthcare reporting for HMPs, THMPs and NRs has decreased during the period of the present study.

Besides the differences of included products, another important difference compared with the study by Jacobsson et al. [3] is the definition of an adverse reaction. In July 2012, a new European pharmacovigilance legislation came into effect [9]. Since then, the definition of an adverse reaction was changed to include not only a response to a medicinal product, which is noxious and unintended at normal doses, but also from medication errors and uses outside the terms of the marketing authorisation (off-label use), including misuse and abuse of the medicinal product.

The last important difference between the present study and the previous one stems from the fact that in addition to reporting by healthcare professionals, pharmacists and patients may also report adverse reactions. These novel reporting routes have been implemented in Sweden since the previous study by Jacobsson et al. [3] and the impact of patient reporting of ADRs related to HMPs is discussed later in this report.

Comparisons between the previous and the present publication could very roughly be viewed as a comparison between the safety profile of all CAM products (previous study) and medicinal products, which have been assessed with respect to quality, safety and efficacy before they are placed on the market, i.e. medicinal products (present study). In contrast to the previous publication, which reported five fatalities, where the causality between the ADRs and the CAM substance was assessed as at least possible, the present study using the same causality criteria contains no fatal adverse reactions. This finding is not surprising because herbal preparations in (T)HMPs have been in clinical use for many years before the approval of the products, allowing time for ADRs to be acknowledged and assessed. In addition, post-approval monitoring of approved medicinal products reduces the risk that self-care products with severe safety problems remain on the market.

For all medicinal products, underreporting of ADRs is still the major issue in spontaneous reporting, and underreporting could be an alternative explanation for the lack of reports regarding fatalities. It is estimated that only $6 \%$ of all ADRs are reported [10]. Contributing factors for the underreporting of ADRs in the present study may be owing to the misbelief that substances derived from nature are generally safe. We also suspect that the use of herbal selfcare products is not always being communicated by patients to their healthcare professionals. For example, in a 
recent study from Germany concerning elderly patients, only six out of ten patients informed their general practitioner of their CAM use [11]. A similar estimation was made in an American study, where more than half of the participants did not disclose their use of herbal treatments to the healthcare professionals [12].

Today, safety of herbal preparations included in medicinal products is also assessed and harmonised on a European level. After the establishment of a pan European legislation [13], approximately 140 European Union herbal monographs have been published by the European Committee on HMPCs on the European Medicines Agency website (www.ema.europa.eu). In these monographs, the member states have assessed and agreed upon important safety and efficacy information on herbal preparations. The monographs, and other safety information published by the HMPC, facilitate and harmonise the processes of approval and the registration of HMPs in the European Union. The committee has continuously published public statements regarding toxic substances found in herbal treatments, for example, pyrrolizidine alkaloids, thujone and aristolochic acid.

As an example that non-medicinal products can pose a threat to the consumer, a product based on shark liver oil led to nine reports of pulmonary embolism, which became one of the most common serious adverse reactions in the previous publication by Jacobsson et al. [3]. No medicinal products based on shark liver oil are approved in Sweden today, and no cases of pulmonary embolism were reported in the present study.

Many initiatives have been developed with the aim of improving healthcare reporting, such as educational activities, means of assisting the reporter and electronic submission of ADRs. One of the investigated initiatives is feedback to the reporter [10]. We believe that publication of assessed data from national competent authorities is one way to convey positive feedback and inspire reporting. It is our hope that the present study will be a reminder to healthcare professionals to discuss self-care products with patients, and to bear in mind that also HMPs, THMPs and NRs can cause ADRs.

\section{Conclusions}

This report from the MPA aims to investigate, summarise and discuss ADR reports related to HMPs, THMPs, and NRs on the Swedish market. In agreement with other studies in the field, adverse reactions related to the skin and subcutaneous tissue were the most commonly reported reactions. Importantly, no previously unknown safety problems were discovered. Adverse drug reaction reports from patients and healthcare professionals are a powerful tool to ensure the safe use of HMPs post-approval, and healthcare professionals and patients are therefore encouraged to report suspected adverse reactions related to HMPs.

Acknowledgements The authors acknowledge Per Claeson, Senior Expert, Topi Laajoki, M.D., and Björn Zethelius, Scientific Director, all at the Medical Products Agency, Uppsala, Sweden, for valuable comments on the manuscript.

Author Contributions E. Svedlund and R. Hägerkvist contributed equally to this article.

\section{Compliance with Ethical standards}

Funding No funding was received for this study.

Conflict of interest Erika Svedlund, Maria Larsson and Robert Hägerkvist have no conflicts of interest directly relevant to the content of this study.

Open Access This article is distributed under the terms of the Creative Commons Attribution-NonCommercial 4.0 International License (http://creativecommons.org/licenses/by-nc/4.0/), which permits any noncommercial use, distribution, and reproduction in any medium, provided you give appropriate credit to the original author(s) and the source, provide a link to the Creative Commons license, and indicate if changes were made.

\section{References}

1. Läkemedelsverkets föreskrifter (LVFS 2006:11) om godkännande av läkemedel för försäljning m.m. (Swedish only). 2006 Elanders, Vällingby, Sweden: www.lakemedelsverket.se. Accessed 1 Sept 2016.

2. Claeson P. Requirements on efficacy of herbal medicinal products. J Ethnopharmacol. 2014;158:463-6. doi:10.1016/j.jep.2014. 07.017.

3. Jacobsson I, Jonsson AK, Gerden B, Hagg S. Spontaneously reported adverse reactions in association with complementary and alternative medicine substances in Sweden. Pharmacoepidemiol Drug Saf. 2009;18(11):1039-47. doi:10.1002/pds.1818.

4. Gardiner P, Adams D, Filippelli AC, Nasser H, Saper R, White L, et al. A systematic review of the reporting of adverse events associated with medical herb use among children. Glob Adv Health Med Improv Healthc Outcomes Worldw. 2013;2(2): 46-55. doi:10.7453/gahmj.2012.071.

5. Farah MH, Edwards R, Lindquist M, Leon C, Shaw D. International monitoring of adverse health effects associated with herbal medicines. Pharmacoepidemiol Drug Saf. 2000;9(2):105-12. doi:10.1002/(SICI) 1099-1557(200003/04)9:2<105:AID-PDS486 $>3.0 . \mathrm{CO} ; 2-2$.

6. European Community on Cimicifuga racemosa (L.) Nutt., rhizoma (EMA/HMPC/600717/2007 Corr. $^{l}$ ) http://www.ema. europa.eu/docs/en_GB/document_library/Herbal_-_Community_ herbal_monograph/2011/01/WC500100981.pdf. Accessed 02 March 2017.

7. Assessment report on Cimicifuga racemosa (L.) Nutt., rhizoma (EMA/HMPC/3968/2008) http://www.ema.europa.eu/docs/en GB/document_library/Herbal_-_HMPC_assessment_report/2011/ 05/WC500106358.pdf. Accessed 02 March 2017. 
8. Danan G, Teschke R. RUCAM in drug and herb induced liver injury: the update. Int J Mol Sci. 2016;17:14. doi:10.3390/ ijms 17010014.

9. Directive 2010/84/EU of the European Parliament and of the Council of 15 December 2010 amending, as regards pharmacovigilance, Directive 2001/83/EC on the Community code relating to medicinal products for human use., Official Journal L 348, 31/12/2010, p. 74-99.

10. Gonzalez-Gonzalez C, Lopez-Gonzalez E, Herdeiro MT, Figueiras A. Strategies to improve adverse drug reaction reporting: a critical and systematic review. Drug Saf. 2013;36(5):317-28. doi:10.1007/s40264-013-0058-2.
11. Schnabel K, Binting S, Witt CM, Teut M. Use of complementary and alternative medicine by older adults-a cross-sectional survey. BMC Geriatr. 2014;14:38. doi:10.1186/1471-2318-14-38.

12. Gardiner P, Graham R, Legedza AT, Ahn AC, Eisenberg DM, Phillips RS. Factors associated with herbal therapy use by adults in the United States. Altern Ther Health Med. 2007;13(2):22-9.

13. Directive 2004/24/EC of the European Parliament and of the Council of 31 March 2004 amending, as regards traditional herbal medicinal products, Directive 2001/83/EC on the Community code relating to medicinal products for human use. Off $\mathbf{J}$ Eur Commun L 136: 85-90. 